\title{
MINIMUM ASSET AND LIABILITY INSURANCE REQUIREMENTS ON JUDGMENT-PROOF INDIVIDUALS WHEN HARM IS ENDOGENOUS*
}

\author{
Chulyoung Kim \\ School of Economics, Yonsei University \\ Seoul 03722, Korea \\ chulyoung.kim@gmail.com \\ and \\ PaUl S. KoH \\ Department of Economics, Columbia University \\ New York 10027, USA \\ paulkoh9@gmail.com
}

Received January 2019; Acceted June 2019

\begin{abstract}
Shavell (2005) studied the optimality of minimum asset requirements within a frame-work in which individuals can influence the probability of an accident. We reinvestigate Shavell's model for the opposite accident scenario, in which individuals can influence the magnitude of harm, and find different policy implications. In particular, we show that it could be optimal to completely ban judgment-proof individuals from participating in a potentially harmful activity. We also examine the effect of liability insurance, and find that regulatory authorities should tighten standards relative to the pure asset requirement and that liability insurance increases social welfare.
\end{abstract}

Keywords: minimum asset requirement, liability insurance, judgment proof, endogenous harm JEL Classification Codes: G28, K13, K20, L51

\section{Introduction}

Regulatory authorities have long been aware of the possibility that actors in hazardous activities may go bankrupt due to their insufficient levels of asset, in which case their incentive to exert enough care in operating their businesses tends to be weak. ${ }^{1}$ This could potentially pose

\footnotetext{
* We are grateful to an anonymous referee and participants at various seminars for their valuable comments. All remaining errors are our own.

1 See U.S. Government Accountability Office (2005) "BLM Needs to Better Manage Financial Assurances to Guarantee Coverage of Reclamation Costs", Report to the Ranking Minority Member, Committee on Homeland
} 
a large threat for our society as improperly handled hazardous wastes could bring us a devastating result, incurring billions of dollars nationwide (Boyd (2001)). In response to this issue, regulatory agencies often require individuals participating in potentially harmful activities such as driving, construction, and transportation of hazardous wastes to maintain a certain level of assets as a precondition for their permits for operation. As their assets are at stake, the actors in hazardous activities have stronger incentive to comply with the law and manage the risk in operating their businesses, as argued by the proponents of such mandatory financial requirements (see Abraham (2010), Jerry and Richmond (2012), and Dobbyn and French (2016)). Although this type of regulation, called minimum asset requirement, is commonly associated with automobiles, it is frequently adopted in other domains as well, including hazardous waste, construction, and banking operation. ${ }^{2}$

Shavell (2005) formally studied the issues related to minimum asset requirements when some individuals are judgment proof. ${ }^{3}$ He provided intuitive conditions on the optimal asset requirement, and studied the effect of liability insurance on individual behavior and social welfare when asset requirements were in effect. His analysis adopted the standard model in the literature in which an individual's precautionary choices influence the probability of an accident but not the magnitude of harm from the accident should it occur; this model is a description of many realistic accident situations such as driving and construction. However, there are other situations in which an individual's influence on the likelihood of an accident is limited whereas the magnitude of harm from the accident crucially depends on the level of precaution taken, and it is not clear if Shavell's results would hold in this type of accident scenario. Indeed, as shown by Dari-Mattiacci and De Geest (2005), individual incentives and behavior are quite different between these two accident scenarios, and therefore they could generate different policy implications for asset requirements. ${ }^{4}$ To study this issue, in this paper, we reinvestigate Shavell's model with the assumption that an individual can influence the magnitude of harm from an accident but not the likelihood of the accident occurring.

In Section II, we first characterize the optimal minimum asset requirement, showing that there exists an asset threshold that divides the total population into two groups: individuals with higher assets who exhibit compliant behavior, thereby making socially optimal precaution and engagement decisions, and individuals with lower assets who exhibit noncompliant behavior, thereby exerting no precaution whatsoever and engaging in the potentially harmful activity too often from the social point of view. We show that such stark behavior adopted by individuals produces policy implications that are different from those found by Shavell. In particular, when

Security and Governmental Affairs, U.S. Senate No. GAO-05-377, Washington, D.C., Jun.

${ }^{2}$ For instance, the Resource Conservation and Recovery Act (RCRA), which is one of major U.S. environmental statutes, legally mandates the principal of financial responsibility to deter environment hazards. See also Fla. Stat. Ch. 403.724 (2016) for financial responsibility in hazardous waste operation, and Wis. Admin. Code $§ 5.31$ (2016) for financial responsibility in construction.

${ }^{3}$ The first study to examine the incentive effects of judgment-proof problems in a formal model was that of Shavell (1986). Whereas Shavell assumed that precaution incurs a nonmonetary utility cost, Beard (1990) considered a model in which precaution incurs a monetary cost. See Miceli and Segerson (2003) for a comparison of these two approaches to the judgement-proof problem. See also Summers (1983), Landes and Posner (1984), Boyd and Ingberman (1994, 1999), and Lewis and Sappington (1999).

${ }^{4}$ Dari-Mattiacci and De Geest (2005) were the first to differentiate these different accident scenarios, and their results are applied in various contexts; see, for instance, Dari-Mattiacci and De Geest (2006), De Geest and Dari-Mattiacci (2007), Dari-Mattiacci and Mangan (2008), and Dari-Mattiacci and Langlais (2012). 
noncompliant behavior imposes substantial losses on society, it could be optimal for the regulatory authority to completely ban noncompliant individuals by allowing only wealthy individuals who are willing to abide by the socially optimal behavior. This is in contrast to the finding of Shavell, who argued that society should always allow some marginally judgmentproof individuals to participate in the harmful activity.

In Section III, we introduce liability insurance to our basic model. Although pure asset requirements are often used in practice, a more widely adopted policy measure is a financial responsibility requirement under which individuals with insufficient assets can still engage in the activity if they can satisfy the requirement by purchasing sufficient insurance coverage. ${ }^{5}$ Thus, it is important to know how a regulatory authority should revise its standards when including insurance coverage in the requirement, and whether liability insurance may improve social welfare. We show that the regulatory authority should tighten the standard when including insurance coverage in the requirement: that is, the optimal financial responsibility requirement should be higher than the optimal pure asset requirement. We also show that, as found by Shavell, social welfare is greater under the optimal financial responsibility requirement in the absence of insurance-related moral hazard, which assures us that liability insurance tends to improve a judgment-proof individual's precaution and engagement decisions under two radically different accident scenarios.

Our paper contributes to the literature on policies and regulations that improve a judgmentproof individual's incentive and behavior. Since the early contributions by Summers (1983), Landes and Posner (1984), and Shavell (1986), scholars have discussed and proposed various policy measures to alleviate the adverse consequences that arise from the judgment-proof problem: for instance, departing from compensatory damages may improve a judgment-proof individual's behavior; ${ }^{6}$ lowering negligence standards for judgment-proof individuals has been shown to increase their precaution levels ${ }^{7}$ and the imposition of vicarious liability, under the doctrine of respondeat superior, on parent firms or lenders could induce efficient behavior. ${ }^{8}$ In this vain, Shavell (2005) analyzed the benefits and costs of using minimum asset requirements, which have been extensively used among policy makers in practice. However, despite the practical importance of minimum asset requirements as a remedy to judgment-proof problems, to the best of our knowledge they have not been formally studied since Shavell's contribution. ${ }^{9}$

\footnotetext{
${ }^{5}$ In practice, many statutes essentially include insurance coverage when specifying the requirements that need to be satisfied by participating individuals. For instance, Florida Statutes Chapter 403.724 (2016) specifies that "cash, the establishment of a trust fund, surety bonds, a letter of credit, or casualty insurance, a financial test, a corporate guarantee, or a combination thereof, may be used to satisfy the financial responsibility requirement."

${ }^{6}$ See, for instance,Boyd and Ingberman $(1994,1999)$ and Lewis and Sappington (1999) for arguments that punitive damages, rather than compensatory damages, may improve a judgment-proof individual's choice because he pays more to victims when actual harm is small. See also Dari-Mattiacci and De Geest (2005) for an argument for using average compensation, which requires less information for damage calculations.

7 See Ganuza and Gómez $(2008,2011)$ and the reference therein. The literature on tort law generally identifies negligence rules as superior to strict liability in unilateral accident settings with judgment-proof injurers. However, in bilateral accident settings, Friehe (2008) showed that strict liability with a defense of contributory negligence can perform better than simple negligence or negligence with a defense of contributory negligence.

${ }^{8}$ See, for example, Sykes (1984), Boyer and Laffont (1997), Hiriart and Martimort (2006), and Bisso and Choi (2008).

${ }^{9}$ Remotely related is the study by Pitchford (1995) who considers an asset requirement as a means of inducing investors to increase equity in potentially judgment-proof firms. Kahane (1977), Kim and Santomero (1988), Rochet
} 
Our paper contributes to this literature by suggesting policy implications about asset and liability insurance requirements under a radically different accident scenario.

Our paper is also related to the literature on the usage of compulsory liability insurance as a way of ameliorating the judgment-proof problem. In line with previous works, ${ }^{10}$ we show that the existence of insurance policies generally improves a judgment-proof individual's behavior in the absence of insurance-related moral hazard.

The remainder of this paper is organized as follows. Section II describes our basic model and studies minimum asset requirements. Section III introduces liability insurance to our basic model, and compares pure asset requirements and financial responsibility requirements. Finally, Section IV offers concluding remarks. All mathematical proofs are in the Appendix.

\section{Minimum Asset Requirements}

We consider a regulatory situation in which a unit mass of risk-neutral individuals may choose to engage in a potentially harmful activity. In this section we formally describe this situation with the following variables:

- $g \geq 0$ : the gain to an individual from engaging in the activity with a continuous density $f(g)>0$ on $\mathbb{R}_{+}$,

- $\mathrm{x} \geq 0$ : the level of care exercised by an individual, ${ }^{11}$

- $p \in(0,1)$ : the probability of an accident,

- $h(x) \geq 0$ : the magnitude of harm in the event of an accident, where $h^{\prime}<0$ and $h^{\prime \prime}>0$, and

- $y \geq 0$ : the level of assets of an individual with a continuous density $w(y)>0$ on $\mathbb{R}_{+}$.

The main difference from the model analyzed in the literature is that the probability of an accident is fixed while the magnitude of harm is endogenous and influenced by the individual's precaution effort. It should be noted that in our framework the judgment-proof problem is inevitable as long as the maximum magnitude of harm, $h(0)$, is sufficiently large: an individual can strategically choose a low level of care leading to $h(x)>y$, subjecting himself to be judgment proof in the event of an accident.

If an individual engages in the activity, the social gain is the individual's private benefit $g$, and the social cost is the sum of the individual's precaution cost $x$ and the expected cost of harm $p h(x)$. Thus, social welfare is the integral of $g-(x+p h(x))$ over all individuals who engage in the activity. To maximize social welfare, each participating individual must choose $x$ so as to minimize the social cost: that is, he must choose $x$ such that

$$
1=-p h^{\prime}(x) \text {. }
$$

Let us denote the first-best precaution level by $x^{*}$, which we assume to be positive. Therefore, to maximize social welfare, an individual must engage in the activity only when his private gain exceeds the minimized social cost:

(1992), and Freixas and Rochet (2008) studied bank solvency regulation as a device for reducing the risk of bank failure.

${ }^{10}$ See, for instance, Jost (1996), Polborn (1998), Shavell (2000), and Cohen and Dehejia (2004).

${ }^{11}$ Following Shavell (2005), an individual's precautionary effort $x$ is assumed to be a nonmonetary utility cost. 


$$
g \geq x^{*}+p h\left(x^{*}\right)
$$

When does an individual have the incentive to abide by the socially optimal behavior prescribed above? Assuming strict liability, an individual's expected cost from engaging in the activity is

$$
\min _{x} x+p \cdot \min \{y, h(x)\} .
$$

Let us denote the solution of this problem as $x(y)$. An individual with asset $y$ will choose $x(y)$ upon engagement, bearing the expected cost of $x(y)+p \cdot \min \{y, h(x(y))\}$, and therefore he will choose to engage in the activity if his private gain is greater than his expected cost:

$$
g \geq x(y)+p \cdot \min \{y, h(x(y))\} .
$$

The following lemma shows that the individual's behavior may not be socially optimal if $y$ is small.

Lemma 1. If an individual's asset $y$ is sufficiently high, that is, if

$$
y \geq h\left(x^{*}\right)+x^{*} / p \equiv \bar{y},
$$

his engagement and precaution decisions are socially optimal. Otherwise, he takes no precaution at all upon engagement and may engage in the activity when it is socially undesirable.

This finding is illustrated in Figure 1. The shaded area denoted by compliance represents the individuals who participate in the activity and exert optimal care. These individuals bear the full costs of an accident under optimal care, and therefore their engagement decision is optimal. By contrast, the blue area denoted by noncompliance represents the individuals who engage in the activity but exert no effort whatsoever. Although these individuals impose social costs of $p h(0)$, their private costs are only $p y$ : their choice of effort subjects themselves to be judgment proof in the event of an accident, in which case they just forfeit their entire assets, thereby leaving victims partially uncompensated for accident losses. Thus, these noncompliant individuals engage in the activity as long as their private gains are higher than their private costs, $g \geq p y$, exhibiting suboptimal engagement decisions. The rest of the population, denoted by no engagement, represents the individuals who do not engage in the activity because their

Figure 1. Individual Behavior

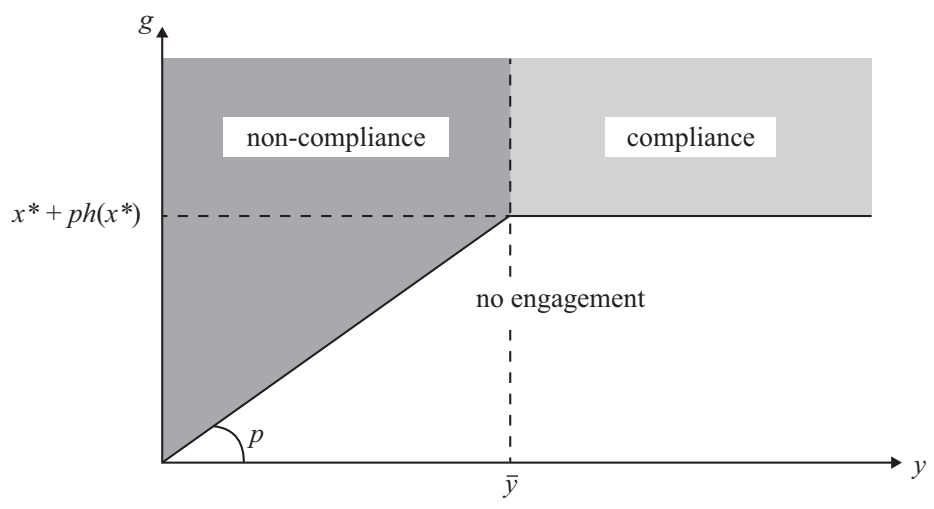


gains are insufficient to cover their costs.

This lemma shows that an individual's behavior is not socially optimal if his asset $y$ is small. First, poor individuals have less incentive to exert care because they bear only a part of the harm incurred from their activities. Second, they may engage in the activity when it is socially undesirable: this happens because their private cost is lower than the social cost from their activities, thereby inducing them to engage in the activity too often from the social point of view.

In contrast to the findings of Shavell who showed $x$ to be a continuous function of $y$, we find that an individual's choice of $x$ is stark, either $x=0$ or $x=x^{*}$, which is essentially the result obtained by Dari-Mattiacci and De Geest (2005). Such noncompliant behavior of poor individuals makes the activity particularly harmful for society when the magnitude of harm is endogenous because the individuals with $y<\bar{y}$ exercise no precaution whatsoever, thereby imposing the maximum possible harm on society in the event of an accident. Moreover, the asset threshold that divides the judgment-proof and solvent groups differs radically between the two approaches. Whereas Shavell showed that such an asset threshold is equal to the magnitude of harm that is exogenously fixed, the threshold in our endogenous-harm framework is given by $\bar{y}=h\left(x^{*}\right)+x^{*} / p$, which is determined endogenously in the model.

When the noncompliant behavior of poor individuals incurs substantial social losses, the judgment-proof problem could be ameliorated by using appropriate regulatory measures that deter individuals who should not engage in the potentially harmful activity. To this end, suppose that the regulatory authority can regulate entry into the activity by use of an asset requirement, and therefore individuals cannot engage in the activity unless their assets are at least $\underline{y}$.

Given an individual's decision rule from Lemma 1, social welfare as a function of the asset requirement $\underline{y}$ can be written as

$$
\begin{aligned}
S \underline{\underline{y}})= & \int_{\bar{y}}^{\infty}\left\{\int_{x^{*}+p h\left(x^{*}\right)}^{\infty}\left(g-\left(x^{*}+p h\left(x^{*}\right)\right)\right) f(g) d g\right\} w(y) d y \\
& +\int_{\underline{y}}^{\bar{y}}\left\{\int_{p y}^{\infty}(g-p h(0)) f(g) d g\right\} w(y) d y
\end{aligned}
$$

where the first expression is the social welfare from compliance, and the second expression is the social welfare from noncompliance. As the individuals with $y \geq \bar{y}$ make socially optimal decisions, it is straightforward to find that the optimal asset requirement, denoted by $\underline{y}^{*}$, cannot exceed $\bar{y}$.

Lemma 2. $\underline{y}^{*} \leq \bar{y}$.

For the following analysis, it is convenient to define a continuous and increasing function $v(k) \equiv E[g \mid g \geq p k]$ for $k \in[0, \bar{y}]$ with $\underline{v} \equiv v(0)$ and $\bar{v} \equiv v(\bar{y})$. This function represents the average gain for the individuals engaging in the activity from the asset group with $y=k$ : if an individual has asset $k \leq \bar{y}$, he bears private costs $p k$ upon engagement, and therefore he participates in the activity if and only if $g \geq p k$. 
To understand the benefit and cost of imposing an asset requirement, from (1) we have for $\underline{y}<\bar{y}$ :

$$
\begin{aligned}
S^{\prime}(\underline{y}) & =\left(\int_{p \underline{y}}^{\infty}(p h(0)-g) f(g) d g\right) w(\underline{y}) \\
& =\left(\int_{p \underline{y}}^{p h(0)}(p h(0)-g) f(g) d g-\int_{p h(0)}^{\infty}(g-p h(0)) f(g) d g\right) w(\underline{y})
\end{aligned}
$$

where the first part inside the parentheses represents the benefit from excluding the low-gain individuals who should not engage, and the second part represents the cost of excluding the high-gain individuals who should have engaged. Thus, the optimal minimum asset requirement should balance these marginal benefits and marginal costs, as the following proposition demonstrates.

Proposition 1. There exists a unique optimal minimum asset requirement $\underline{y}^{*}$. More precisely:

(1) interior solution: $\underline{y}^{*} \in(0, \bar{y})$ such that $p h(0)=v\left(\underline{y}^{*}\right)$ if and only if $p h(0) \in(\underline{v}, \bar{v})$

(2) corner solution: (i) $\underline{y}^{*}=0$ if and only if $p h(0) \leq \underline{v}$ and (ii) $\underline{y}^{*}=\bar{y}$ if and only if $p h(0) \geq \bar{v}$

We provide explanations for the proposition in the following with three corollaries. When the expected harm from noncompliance is relatively small (i.e., $p h(0) \leq \underline{v}$ ), it is desirable to impose no restriction on entry to the activity (i.e., $\underline{y}^{*}=0$ ). However, as the noncompliant behavior becomes detrimental (i.e., $p h(0)$ increases), society can benefit from excluding the poorest individuals by setting $\underline{y}^{*}>0$ because they are likely to choose to participate even when their gains from the activity are negligible. Thus, the necessary and sufficient condition for regulating entry (i.e., $\underline{y}^{*}>0$ ) to be socially desirable is that the expected harm from noncompliance is greater than the average gain accruing to the poorest (i.e., $\left.p h(0)>_{\underline{v}}\right)$. This observation from the proposition is summarized in the following corollary.

Corollary 1. $\underline{y}^{*}>0$ if and only if $p h(0)>\underline{v}$.

Shavell obtained an analogous condition in his setting because, in essence, the behavior of the poorest is the same in his model and our model: the individual with no asset bears no private cost upon engagement in the activity, and therefore he is always willing to participate in the activity without exerting any effort.

When the expected harm from noncompliance is moderate (i.e., $p h(0) \in(\underline{v}, \bar{v})$ ), we obtain an interior solution characterized by

$$
\operatorname{ph}(0)=v\left(\underline{y}^{*}\right)
$$

which shows that $\underline{y}^{*}$ increases as $p h(0)$ increases because $v(\cdot)$ is an increasing function. This observation from the proposition is summarized in the following corollary.

Corollary 2. $\underline{y}^{*}$ increases as ph(0) increases as long as $p h(0) \in(\underline{v}, \bar{v})$.

The right-hand side of equation (2) is 


$$
v\left(\underline{y}^{*}\right)=E\left[g \mid g \geq p \underline{y}^{*}\right]
$$

which represents the average gain from the individuals (with asset $\underline{y}^{*}$ ) participating in the activity. The left-hand side is the expected harm from noncompliance. Thus, social welfare from allowing the individuals with asset $\underline{y}^{*}$ is 0 because social gains and losses are the same according to equation (2). Then, it follows that social welfare from allowing the individuals with asset $y<\underline{y}^{*}$ (respectively, $y>\underline{y}^{*}$ ) is negative (respectively, positive) because $v(\cdot)$ is an increasing function. That is, while social losses represented by $p h(0)$ are constant, social gains represented by $v(y)$ is lower than $v\left(\underline{y}^{*}\right)$ for $y<\underline{y}^{*}$ and higher than $v\left(\underline{y}^{*}\right)$ for $y>\underline{y}^{*}$. This is why the optimal minimum asset requirement allows the individuals only with asset higher than or equal to $y^{*}$.

As the expected harm from noncompliance increases, the regulatory authority seeks to increase the asset requirement, banning more asset groups from engaging in the activity. One particular group of individuals deserves a specific mention: the individuals with asset $y=h\left(x^{*}\right)$. Some individuals with asset $y=h\left(x^{*}\right)$ are in the noncompliance zone because $h\left(x^{*}\right)<\bar{y}$. ${ }^{12}$ Thus, if social welfare from their engagement is negative, it is socially desirable to prevent them from engaging in the activity. This observation from the proposition is summarized in the following corollary. ${ }^{13}$

Corollary 3. $\underline{y}^{*}>h\left(x^{*}\right)$ if and only if $p h(0)>v\left(h\left(x^{*}\right)\right)$.

Shavell showed that the optimal minimum asset requirement should be always smaller than the magnitude of accident harm, but Corollary 3 describes a different picture in our framework. Suppose the regulatory authority estimates that the magnitude of harm from compliance (i.e., $h\left(x^{*}\right)$ ) is one million dollars. With this information about the level of harm from hazardous activities, if the authority follows the advice from Shavell, it should set the minimum asset requirement lower than one million dollars. In contrast, Corollary 3 suggests that the authority may have to set the minimum asset requirement higher than one million dollars under some conditions. This finding suggests that the nature of accident problem (i.e., whether the magnitude of harm is exogenous as in Shavell's model or endogenous as in our model) is crucial in setting the optimal minimum asset requirement.

As a final remark about Proposition 1, if the expected harm from noncompliance is very large, the regulatory authority may seek to exclude all noncompliance asset groups from engaging in the activity, permitting only compliance groups to participate. This is in contrast to Shavell's finding. He argued that the regulatory authority should always allow some judgmentproof individuals to participate in the activity: as individual precaution is continuous in asset when the magnitude of harm is exogenous, marginally lowering the asset requirement from the

\footnotetext{
12 Observe that, as can be seen in Figure 1, some individuals with asset $y=h\left(x^{*}\right)$ are in the noncompliance zone and the remaining individuals with asset $y=h\left(x^{*}\right)$ are in the no-engagement zone.

${ }^{13}$ Corollary 3 follows immediately from the proof of Proposition 1. In general, Corollary 3 can be stated for any $k<\bar{y}$ as: $\underline{y}^{*}>k$ if and only if $p h(0)>v(k)$. To see this, from (7) in page 16, we have $S^{\prime}(k)>0$ if and only if $p h(0)>v(k)$ for any $k<\bar{y}$. This implies that $\underline{y}^{*}$ is larger than $k$ because social welfare is increasing at $k$.
} 
level of harm, and thereby allowing marginally judgment-proof individuals to participate, causes no first-order loss in social welfare because incentives for the newly admitted individuals are essentially optimal, whereas their engagement generates positive first-order gains in social welfare. This is not true in our endogenous-harm framework: as all judgment-proof individuals exert no precaution whatsoever, thereby imposing the maximum possible harm on society, their engagement causes first-order losses in social welfare. Thus, it could be socially preferable to allow engagement only by wealthy individuals who are willing to comply with the socially optimal behavior.

These findings suggest that the endogeneity of accident harm has important policy implications for using minimum asset requirements, which, to the best of our knowledge, have not previously been addressed in the literature. In the next section, we examine the optimal choice of regulatory standards when individuals with insufficient assets are allowed to satisfy the requirement by purchasing insurance coverage.

\section{Minimum Asset and Liability Insurance Requirements}

In this section, we examine how regulatory standards should be changed when individuals are allowed to satisfy the requirement by purchasing insurance coverage. To this end, we introduce additional variables as follows:

- $\underline{z} \geq 0$ : the financial responsibility requirement,

- $c \geq 0$ : insurance coverage in the event of an accident, and

- $\pi \geq 0$ : the insurance premium where $\pi=p c$ (i.e., insurance products are actuarially fair). ${ }^{14}$

Under a financial responsibility requirement $\underline{z}$, a poor individual with $y<\underline{z}$ can still participate in the activity if he buys sufficient insurance coverage to satisfy the following inequality:

$$
y-\pi+c \geq \underline{z} .
$$

Assuming that insurance products are actuarially fair (i.e., $\pi=p c$ ), the inequality above can be reduced to

$$
y+(1-p) c \geq \underline{z} .
$$

As an individual's budget is limited to his asset, the maximum insurance coverage that can be bought is $c=y / p$, which is the amount of insurance when the individual spends all his asset on buying insurance coverage. Thus, the aforementioned inequalities imply that an individual with $y<\underline{z}$ can still engage in the activity if $y \geq p \underline{z} \cdot{ }^{15}$ This observation implies that a financial

\footnotetext{
${ }^{14}$ In our formulation, observe that the insurance-related moral hazard problem does not exist because the probability of an accident is fixed at $p$ regardless of the level of precaution chosen by an individual.

${ }^{15}$ More precisely, if an individual spends all his asset on buying insurance coverage, we have $y=\pi$ and $c=y / p$. Thus, we have

$$
y-\pi+c=y / p \geq \underline{z} \Longleftrightarrow y \geq p \underline{z} .
$$
}


responsibility requirement tends to allow more individuals to engage in the activity than an asset requirement.

In the following analysis, we demonstrate that the optimal financial responsibility requirement, $\underline{z}^{*}$, is higher than the optimal minimum asset requirement, $\underline{y}^{*}$, and that the former improves social welfare relative to the latter. To this end, we first state our basic findings about an individual's behavior under a financial responsibility requirement in the following lemma.

Lemma 3. There are three types of individuals depending on their levels of asset: $y<p \underline{z}$, $y \in[p \underline{z}, \underline{z})$, and $y \geq \underline{z}$.

1. The individuals with assets $y \geq \underline{z}$ will not buy insurance coverage when engaging in the activity; if they engage in the activity, they choose $x=x^{*}$ if $y \geq \bar{y}$, and $x=0$ otherwise.

2. The individuals with assets $y \in[p \underline{z}, \underline{z})$ will buy the minimum necessary coverage $c>0$ such that $y-\pi+c=\underline{z}$ when engaging in the activity; if they engage in the activity, they choose $x=x *$ if $z \geq \bar{y}$, and $x=0$ otherwise.

3. The individuals with assets $y<p \underline{z}$ cannot engage in the activity.

If the regulatory authority imposes the strictest requirement, $\underline{z}=\bar{y}$, all asset groups eligible for engagement make socially optimal decisions. According to Lemma 3, the individuals with $y \geq \bar{y}$ (i) do not purchase insurance coverage, (ii) exert optimal precaution upon engagement, and (iii) engage if and only if $g \geq x^{*}+p h\left(x^{*}\right)$. The individuals with $y \in[p \bar{y}, \bar{y})(i)$ purchase insurance coverage such that $y-\pi+c=\bar{y}$, (ii) exert optimal precaution upon engagement, and (iii) engage if and only if $g \geq x^{*}+p h\left(x^{*}\right)$. The rest of the population cannot participate because they cannot satisfy the financial responsibility requirement. This situation is illustrated in Figure 2. Observe that all individuals are either in the compliance zone or in the no-engagement zone. Thus, social welfare under $\underline{z}=\bar{y}$ is

Figure 2. Individual BeHAVIOR UNDER $\underline{z}=\bar{y}$

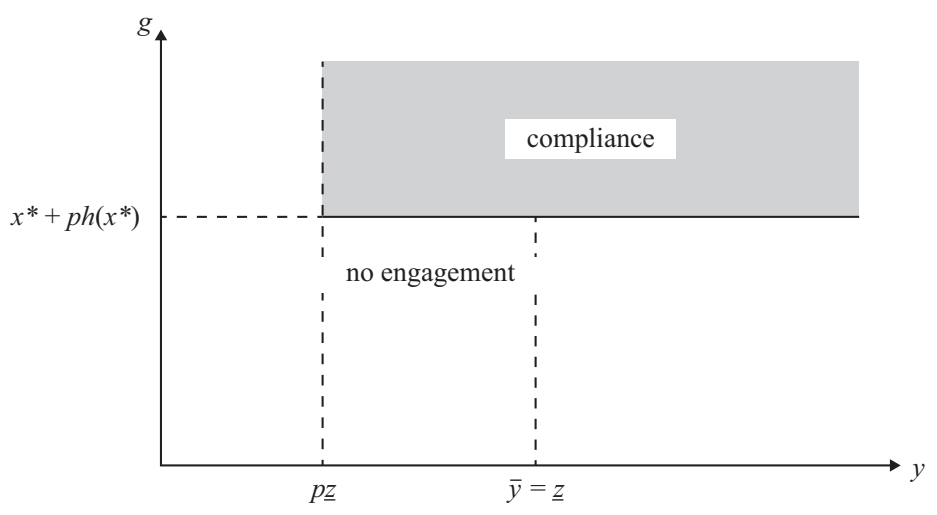




$$
T(\bar{y})=\int_{p y}^{\infty}\left\{\int_{x^{*}+p h\left(x^{*}\right)}^{\infty}\left(g-\left(x^{*}+p h\left(x^{*}\right)\right)\right) f(g) d g\right\} w(y) d y .
$$

If the regulatory authority adopts a more lenient requirement, $\underline{z}<\bar{y}$, a rich individual's behavior will be the same according to Lemma 3: the individuals with $y \geq \bar{y}>\underline{z}$ (i) do not purchase insurance coverage, (ii) exert optimal precaution upon engagement, and (iii) engage if and only if $g \geq x^{*}+p h\left(x^{*}\right)$. However, the individuals with $y \in[p \underline{z}, \bar{y})$ exhibit radically different behavior. According to Lemma 3, the individuals with $y \in[\underline{z}, \bar{y})(i)$ do not purchase insurance coverage, (ii) exert no precaution upon engagement, and (iii) engage if and only if $g \geq p y$. The individuals with $y \in[p \underline{z}, \underline{z})(i)$ purchase insurance coverage such that $y-\pi+c=\underline{z}$, (ii) exert no precaution upon engagement, and (iii) engage if and only if $g \geq p \underline{z}$. The rest of the population cannot participate because they cannot satisfy the financial responsibility requirement. This situation is illustrated in Figure 3. Thus, social welfare under $\underline{z}<\bar{y}$ is

$$
\begin{aligned}
T(\underline{z})= & \int_{p \underline{z}}^{z}\left\{\int_{p \underline{\underline{z}}}^{\infty}(g-p h(0)) f(g) d g\right\} w(y) d y+\int_{\underline{z}}^{\bar{y}}\left\{\int_{p y}^{\infty}(g-p h(0)) f(g) d g\right\} w(y) d y \\
& +\int_{\bar{y}}^{\infty}\left\{\int_{x^{*}+p h\left(x^{*}\right)}^{\infty}\left(g-\left(x^{*}+p h\left(x^{*}\right)\right)\right) f(g) d g\right\} w(y) d y .
\end{aligned}
$$

To find $\underline{z}^{*}$, it is sufficient to consider $\underline{z} \leq \bar{y}$ as the following lemma states. To understand this lemma, consider $\underline{z}^{\prime}>\bar{y}$. Then, according to Lemma 3 , the individuals with assets $y \geq p \underline{z}$ make precaution and engagement decisions that are socially optimal, whereas the others with assets $y<p \underline{z}$ cannot engage. Then, reducing $\underline{z}^{\prime}$ to $\underline{z}^{\prime \prime}>\bar{y}$ increases social welfare because the individuals with assets $y \in\left[p \underline{z}^{\prime \prime}, p \underline{z}^{\prime}\right)$ are induced to engage with optimal decisions, and other individuals behave in the same way. Therefore, we must have $\underline{z}^{*} \leq \bar{y}$ as the following lemma states.

Figure 3. Individual BehaVior UNDer $\underline{z}<\bar{y}$

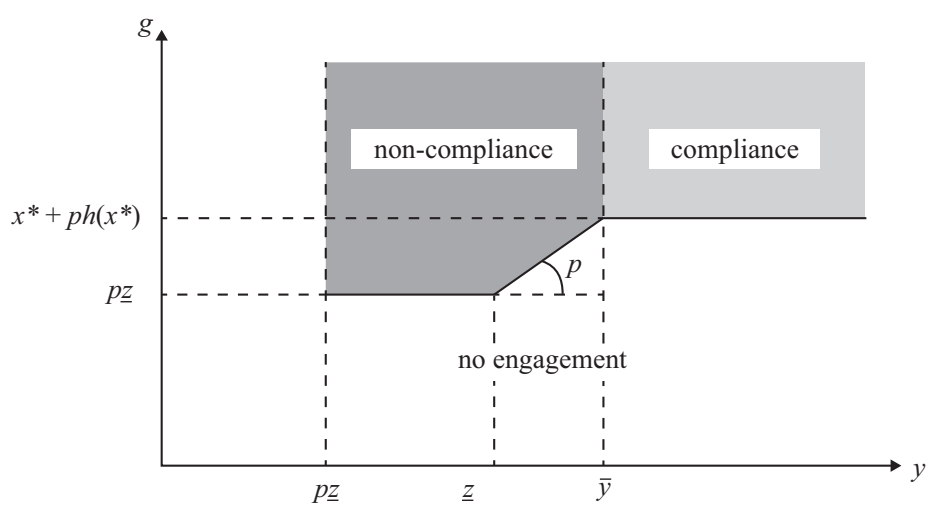


Lemma 4. $\underline{z}^{*} \leq \bar{y}$.

Observe that at $\underline{z}=\bar{y}$, there is a discrete upward jump in social welfare because the individuals with $y \in[p \bar{y}, \bar{y})$, who were in the noncompliance zone under $\underline{z}<\bar{y}$, change their behavior to compliance under $\underline{z}=\bar{y}$. This discontinuity suggests that $\underline{z}=\bar{y}$ could be optimal for a wide range of parameter values, as suggested in the following lemma.

Lemma 5. There exists $\hat{y} \in[0, \bar{y})$ such that $\underline{z}^{*} \notin[\hat{y}, \bar{y})$.

We are now ready to state our main results regarding the comparison of $\underline{z}^{*}$ and $y^{*}$ :

Proposition 2. We have $\underline{y}^{*} \leq \underline{z}^{*}$. More precisely:

1. $0=\underline{y}^{*} \leq \underline{z}^{*}$ if $p h(0) \leq \underline{v}$

2. $0<\underline{y}^{*}<\underline{z}^{*}$ if $p h(0) \in(\underline{v}, \bar{v})$

3. $\bar{y}=\underline{y}^{*}=\underline{z}^{*}$ if $p h(0) \geq \bar{v}$

When the expected harm from noncompliance is negligible, it is socially beneficial not to impose any restriction on entry, under both the pure asset requirement and the financial responsibility requirement. As noncompliance becomes more harmful, under the pure asset requirement the regulatory authority should exclude the asset groups at the bottom of the asset distribution, thus imposing $y^{*}>0$. In this case, we find that the regulatory authority should tighten the requirement, that is, $\underline{z}^{*}>\underline{y}^{*}$, when allowing individuals to satisfy the requirement with insurance coverage. To see the intuition for this finding, observe that marginally increasing $\underline{z}$ at $\underline{z}=\underline{y}^{*}$ has a positive effect on social welfare:

$$
T^{\prime}\left(\underline{y}^{*}\right)=p\left(p h(0)-p \underline{y}^{*}\right) f\left(p \underline{y}^{*}\right) \int_{p \underline{\underline{*}}{ }^{*}}^{\underline{y}^{*}} w(y) d y>0 .
$$

The engagement decisions of the asset groups with $y \in\left(p \underline{y}^{*}, \underline{y}^{*}\right)$ improve under a stricter requirement: because they should purchase greater insurance coverage than before, thereby bearing higher private costs, they choose to engage in the activity when their private gains are larger. Observe that higher deterrence for these asset groups is beneficial: as $p h(0)=v\left(\underline{y}^{*}\right)$ and $v(\cdot)$ is an increasing function, we have $p h(0)>v(y)$ for $y \in\left(p \underline{y}^{*}, \underline{y}^{*}\right)$. Therefore, social welfare from these asset groups increases if $\underline{z}$ increases marginally at $\underline{z}=\underline{y}^{*}$.

This proposition, together with the result from the previous section, provides us with the following information about the lower bound for $z^{*}$. This result follows immediately because the regulatory authority should tighten its standards under a financial responsibility requirement.

Corollary 4. For $k<\bar{y}$, we have $\underline{z}^{*}>k$ if $p h(0)>v(k)$.

Our final result demonstrates that the regulatory authority can improve social welfare by allowing individuals to satisfy the requirement with insurance coverage. 
Proposition 3. We have $S\left(\underline{y}^{*}\right) \leq T\left(\underline{z}^{*}\right)$. More precisely:

1. $S\left(\underline{y}^{*}\right) \leq T\left(\underline{z}^{*}\right)$ if $p h(0) \leq \underline{v}$

2. $S\left(\underline{y}^{*}\right)<T\left(\underline{z}^{*}\right)$ if $p h(0)>\underline{v}$

If the expected harm from noncompliance is negligible, it is beneficial not to impose any restriction on entry to the activity, both under the pure asset requirement and under the financial responsibility requirement. As all individuals behave in the same way when there is no restriction, social welfare is the same in both policy regimes. However, as the noncompliance behavior becomes more harmful to society, the regulatory authority should exclude the individuals at the bottom of the asset distribution by imposing an asset requirement that each participating individual should satisfy. In this case, insurance coverage tends to improve individual behavior: the individuals participating from low asset groups are in effect induced to bear higher private costs by purchasing insurance coverage under the financial responsibility requirement than under the pure asset requirement. Although this effect does not ameliorate the precaution decisions of those individuals, it increases deterrence of these asset groups because they engage in the activity when their private gains are higher than under the pure asset requirement. Thus, the regulatory authority can improve social welfare under the financial responsibility requirement as shown in Proposition 3.

This result, together with Shavell's, assures us that liability insurance tends to improve social welfare regardless of the endogeneity of accident harm when insurance-related moral hazard problems are absent.

\section{Concluding Remarks}

When Shavell (2005) studied the benefits and costs of restricting entry to potentially hazardous activities in the form of either pure asset requirements or financial responsibility requirements, he employed a model in which the probability of an accident is endogenous but the magnitude of harm in the event of an accident is exogenously fixed. We have reinvestigated his findings in a framework in which the size of harm is endogenous and dependent on an individual's precautionary choice in the manner of Dari-Mattiacci and De Geest (2005), and found policy implications that differ from those of Shavell's.

We found that when harm is endogenous, individuals from the lower segment of the asset distribution are induced to exhibit noncompliant behavior: they take no precaution at all, thereby imposing the maximum possible harm on society, and engage in the activity too often from the social point of view. We showed such noncompliant behavior to have important consequences on policy measures. In particular, when the noncompliance behavior is quite dangerous for society, it could be optimal to allow only wealthy individuals who are willing to abide by the socially optimal behavior to engage in the activity. We also examined the consequences of including liability insurance in the requirement, and found that the regulatory authority should tighten its standards relative to the pure asset requirement. Furthermore, we showed that liability insurance increases a judgment-proof individual's private costs, thereby improving his engagement decision, which increases social welfare. 


\section{APPENDIX}

\section{Proof for Lemma 1}

An injurer's cost function, given that he engages in the activity, is

$$
\begin{array}{rrr}
x+p h(x) & \text { if } & h(x) \leq y \\
x+p y & \text { if } & h(x)>y .
\end{array}
$$

The first expression is minimized at $x=x^{*}$ and the second at $x=0$. The injurer chooses $x=x^{*}$ if and only if the expected cost of staying solvent is less than the expected cost of becoming judgment proof:

$$
x^{*}+p h\left(x^{*}\right) \leq p y \Longleftrightarrow \bar{y} \leq y .
$$

Otherwise, he chooses $x=0$ upon engagement. ${ }^{16}$

To verify that these results satisfy the conditions given in (5) and (6), observe that (i) $h\left(x^{*}\right) \leq y$ holds for a solvent individual because $h\left(x^{*}\right)<y \leq y$, and (ii) $y<h(0)$ holds for a judgment-proof individual because $y<\bar{y}<h(0)$, where the second inequality follows from

$$
x^{*}+p h\left(x^{*}\right)<0+p h(0) \Longleftrightarrow \bar{y}<h(0) .
$$

Thus, if $y \geq \bar{y}$, the individual chooses $x=x^{*}$ and bears the expected cost of $x^{*}+p h\left(x^{*}\right)$ upon engagement; he therefore chooses to engage in the activity if and only if $g \geq x^{*}+p h\left(x^{*}\right)$, which is socially optimal. If $y<\bar{y}$, the individual chooses $x=0$ and bears the expected cost of $p y$ upon engagement; thus, he may choose to engage in the activity when $g \in[p y, p h(0))$, which is socially undesirable.

\section{Proof for Lemma 2}

This follows immediately from expression (1).

\section{Proof for Proposition 1}

According to Lemma 2, we can restrict our attention to $\underline{y} \in[0, \bar{y}]$. (1) To show sufficiency, suppose $p h(0) \in(\underline{v}, \bar{v})$. Because $v(\underline{y})$ is continuous and strictly increasing in $\underline{y} \in[0, \bar{y}]$, there exists a unique $\underline{y}^{*} \in(0, \bar{y})$ such that $p h(0)=v\left(\underline{y}^{*}\right)$. This $\underline{y}^{*}$ is a unique candidate for the interior optimum because we have from (1)

$$
\begin{aligned}
S^{\prime}(\underline{y}) & =\left(\int_{p \underline{p}}^{\infty}(p h(0)-g) f(g) d g\right) w(\underline{y}) \\
& =\left(\int_{p \underline{p}}^{\infty} p h(0) f(g) d g-\int_{p \underline{y}}^{\infty} g f(g) d g\right) w(\underline{y}) \\
& =(1-F(p \underline{y}))\left(p h(0)-\int_{p \underline{y}}^{\infty} \frac{f(g)}{1-F(p \underline{y})} d g\right) w(\underline{y}) \\
& =(1-F(p \underline{y}))(p h(0)-[g \mid g \geq p \underline{y}]) w(\underline{y})
\end{aligned}
$$

\footnotetext{
${ }^{16}$ Without loss of generality, we assume that an individual exerts the optimal level of precaution when indifferent.
} 


$$
=(1-F(p \underline{y}))(p h(0)-v(\underline{y})) w(\underline{y})
$$

which is equal to 0 if and only if $p h(0)=v(y)$. We can exclude corner solutions because $S^{\prime}(0)>0$ and $S^{\prime}(\bar{y})<0$, and therefore $\underline{y}^{*}$ is a unique candidate for the optimum. To show that $\underline{y}^{*}$ satisfies the second order condition, we have

$$
\begin{aligned}
S^{\prime \prime}\left(\underline{y}^{*}\right)= & -p f\left(p \underline{y}^{*}\right) \underbrace{\left.p h(0)-v\left(\underline{y}^{*}\right)\right)}_{=0} w\left(\underline{y}^{*}\right)-v^{\prime}\left(\underline{y}^{*}\right)\left(1-F\left(p \underline{y}^{*}\right)\right) w\left(\underline{y}^{*}\right) \\
& +\left(1-F\left(p \underline{y}^{*}\right)\right) \underbrace{\left(p h(0)-v\left(\underline{y}^{*}\right)\right)}_{=0} w^{\prime}\left(\underline{y}^{*}\right) \\
= & -v^{\prime}\left(\underline{y}^{*}\right)\left(1-F\left(p \underline{y}^{*}\right)\right) w\left(\underline{y}^{*}\right)
\end{aligned}
$$

which is negative. Thus, $\underline{y}^{*}$ is a unique optimum.

To show necessity, suppose that $\underline{y}^{*} \in(0, \bar{y})$ is a unique optimum such that $p h(0)=v\left(\underline{y}^{*}\right)$. If $p h(0) \leq \underline{v}$, then $p h(0)<v(\underline{y})$ for all $\underline{y} \in(0, \bar{y})$ because $v \underline{y})$ is strictly increasing in $\underline{y}$. This implies that $S^{\prime}(\underline{y})<0$ for all $\underline{y} \in(0, \bar{y})$ from (7), and therefore $\underline{y}^{*} \in(0, \bar{y})$ cannot be the solution. Thus, we must have $p h(0)>\underline{v}$. Similarly, if $p h(0) \geq \bar{v}$, we have $p h(0)>v(\underline{y})$ for all $\underline{y} \in(0, \bar{y})$, implying $S^{\prime}(\underline{y})>0$ for all $\underline{y} \in(0, \bar{y})$ from (7), and therefore $\underline{y}^{*}$ cannot be the solution. Thus, we must have $p h(0)<\bar{v}$.

(2) (i) Suppose $p h(0) \leq \underline{v}$. This implies that $p h(0)<v(\underline{y})$ for all $\underline{y} \in(0, \bar{y}]$. Then we have $S^{\prime}(\underline{y})<0$ for all $\underline{y} \in(0, \bar{y}]$ from (7), and therefore we have $\underline{y}^{*}=0$. To show necessity, suppose $p h(0)>\underline{v}$. Then we have $S^{\prime}(0)>0$ from (7), which is a contradiction to $\underline{y}^{*}=0$. (ii) Suppose $p h(0) \geq \bar{v}$. This implies that $p h(0)>v(\underline{y})$ for all $\underline{y} \in[0, \bar{y})$. Then we have $S^{\prime}(\underline{y})>0$ for all $\underline{y} \in[0, \bar{y})$ from (7), and therefore we have $\underline{y}^{*}=\bar{y}$ because $\underline{y}^{*} \leq \bar{y}$ by Lemma 2 . To show necessity, suppose $p h(0)<\bar{v}$. Then we have $S^{\prime}(\bar{y})<0$ from (7), which is a contradiction to $\underline{y}^{*}=\bar{y}$.

\section{Proof for Lemma 3}

Let $y \geq \underline{z}$ and fix $x$ and $g$. If an individual does not purchase insurance coverage, his expected payoff from engagement is

$$
g+y-x-p \cdot \min \{y, h(x)\} .
$$

If he purchases coverage, his expected payoff is

There are two cases:

$$
g+y-x-p c-p \cdot \min \{y-p c, h(x)-c\} .
$$

1. Suppose $c \leq h(x)$. (1) First, consider the case $y<h(x)$, that is, the individual who does not purchase coverage is judgment proof. Then $(8)=g+y-x-p y$. To determine (9), we need to consider two cases: (i) $y-p c<h(x)-c$ and (ii) $y-p c \geq h(x)-c$. Under (i), we have (9) $=g+y$ $-x-p c-p y+p^{2} c<g+y-x-p y=(8)$. Under (ii), we have (9) $=g+y-x-p c-p h(x)+p c<g$ 
$+y-x-p y=(8)$. Thus if $y<h(x)$, we have (8) $>(9)$ and therefore the individual does not purchase insurance coverage. (2) Second, consider the case $y \geq h(x)$, that is, the individual who does not purchase insurance coverage is solvent. We have $(8)=g+y-x-p h(x)$ and (9) $=g+y-x-p h(x)$ where the second equality holds because $y-p c \geq h(x)-c$. As the individual is indifferent, we can assume that he does not purchase insurance coverage.

2. Suppose $c>h(x)$. Then we have (9) $=g+y-x-p c+p(c-h(x))=g+y-x-p h(x)$ which is smaller than or equal to (8); that is, (8) $>(9)$ when $y<h(x)$ and (8) $=(9)$ when $y \geq h(x)$. Thus the individual does not purchase insurance coverage.

Given that the individual with $y \geq z$ does not purchase insurance coverage when engaging in the activity, that the individual, upon engagement, chooses $x=x^{*}$ if $y \geq \bar{y}$ and $x=0$ otherwise follows from Lemma 1 .

If $y \in[p \underline{z}, \underline{z}$ ), the individual must buy coverage $c>0$ such that $y-\pi+c \geq \underline{z}$ to engage in the activity.

Fixing $g$ and $x$, there are two cases:

1. Suppose $h(x) \leq \underline{z}$. If the individual buys insurance and engages in the activity, his expected payoff is

$$
g-x+(1-p)(y-p c)+p(y-p c+c-h(x))
$$

which is, after rearranging, equal to $g-x+y-p h(x)$. Thus the individual is indifferent regarding the level of $c>0$ as long as it satisfies $y-\pi+c \geq \underline{z}$ and $y \geq \pi$, and therefore we can assume that he will choose $c$ such that $y-\pi+c=\underline{z}$.

2. Suppose $h(x)>\underline{z}$. There are three subcases: (i) If the individual chooses $c>0$ such that $y-p c+c=\underline{z}$, his expected payoff is, after rearranging, equal to $g+y-p \underline{z}-x$. (ii) If the individual chooses $c>0$ such that $y-p c+c \in(\underline{z}, h(x))$, his expected payoff is $g+(1-p)(y-p c)$

$-x$. We have

$$
\begin{aligned}
& \underline{z}<y-p c+c \\
& \Longleftrightarrow g+(1-p)(y-p c)-x<g+y-p \underline{z}-x
\end{aligned}
$$

which shows that the expected payoff under (ii) is lower than that under (i). (iii) If the individual chooses $c>0$ such that $y-p c+c \geq h(x)$, his expected payoff is $g+y-p h(x)-x$. We have $g+y-p h(x)-x<g+y-p \underline{z}-x$ because $h(x)>\underline{z}$, which shows that the expected payoff under (iii) is lower than that under (i). Therefore he will choose $c>0$ such that $y-\pi+c=z$.

Thus if $y \in[p \underline{z}, \underline{z})$, the individual will choose $c>0$ such that $y-\pi+c=\underline{z}$ when engaging in the activity.

Using this result, the individual's expected payoff from buying the minimum insurance coverage and engaging in the activity is

$$
\begin{aligned}
g+y-p h(x)-x & \text { if } \underline{z} \geq h(x) \\
g+y-p \underline{z}-x & \text { if } \underline{z}<h(x)
\end{aligned}
$$

where the first expression is maximized at $x=x^{*}$ and the second at $x=0$. Thus, upon engagement, the individual chooses $x=x^{*}$ if $g+y-p h\left(x^{*}\right)-x^{*} \geq g+y-p \underline{z}$, or equivalently, if $\underline{z} \geq \bar{y}$, and $x=0$ otherwise. The condition for the first expression above, $\underline{z} \geq h\left(x^{*}\right)$, is satisfied because $\underline{z} \geq \bar{y}>h\left(x^{*}\right)$. The condition 
for the second expression above, $\underline{z}<h(0)$, is satisfied because $\underline{z}<\bar{y}<h(0)$. This completes the proof.

If $y<p \underline{z}$, the individual cannot satisfy $\underline{z}$ even after giving up his entire asset for the insurance premium (i.e., $y=\pi=p c$ ), because $y-\pi+c=c=y / p<\underline{z}$. Thus, he cannot engage in the activity.

\section{Proof for Lemma 4}

Consider $\underline{z}^{\prime}>\bar{y}$. According to Lemma 3, the individuals with assets $y \geq p \underline{z}$ make precaution and engagement decisions that are socially optimal, whereas the others with assets $y<p \underline{z}$ cannot engage. Then, reducing $\underline{z}^{\prime}$ to $\underline{z}^{\prime \prime}>\bar{y}$ increases social welfare because the individuals with assets $y \in\left[p \underline{z}^{\prime \prime}, p \underline{z^{\prime}}\right)$ are induced to engage with optimal decisions, and other individuals behave in the same way. Therefore, we have $\underline{z}^{*} \leq \bar{y}$.

\section{Proof for Lemma 5}

From (3) and (4), we have

$$
\begin{aligned}
\lim _{\underline{\underline{z} \rightarrow \bar{y}}} T(\underline{z})= & \int_{p \bar{y}}^{\bar{y}}\left\{\int_{\overline{p y}}^{\infty}(g-p h(0)) f(g) d g\right\} w(y) d y \\
& +\int_{\bar{y}}^{\infty}\left\{\int_{x^{*}+p h\left(x^{*}\right)}^{\infty}\left(g-\left(x^{*}+p h\left(x^{*}\right)\right)\right) f(g) d g\right\} w(y) d y \\
< & \int_{p \bar{y}}^{\bar{y}}\left\{\int_{x^{*}+p h\left(x^{*}\right)}^{\infty}\left(g-\left(x^{*}+p h\left(x^{*}\right)\right)\right) f(g) d g\right\} w(y) d y \\
& +\int_{\bar{y}}^{\infty}\left\{\int_{x^{*}+p h\left(x^{*}\right)}^{\infty}\left(g-\left(x^{*}+p h\left(x^{*}\right)\right)\right) f(g) d g\right\} w(y) d y \\
= & T(\bar{y})
\end{aligned}
$$

which implies that there exists $\hat{y} \in[0, \bar{y})$ such that $T(\underline{z})<T(\bar{y})$ for $\underline{z} \in(\hat{y}, \bar{y})$. Thus, $\underline{z}^{*}$ cannot be in $\left.\hat{y}, \bar{y}\right)$. Without loss of generality, we assume that the regulatory authority prefers $\underline{z}=\bar{y}$ when $T(\hat{y})=T(\bar{y})$. This completes the proof.

\section{Proof for Proposition 2}

First, suppose $p h(0) \leq \underline{v}$. Then, according to Proposition 1 , we have $\underline{y}^{*}=0$. As $\underline{z}^{*} \geq 0$, we have $0=\underline{y}^{*} \leq \underline{z}^{*}$.

Second, suppose $p h(0) \in(\underline{v}, \bar{v})$. Then, according to Proposition 1, we have $\underline{y}^{*} \in(0, \bar{y})$ such that $p h(0)=v\left(\underline{y}^{*}\right)$. From (4), we have

$$
\begin{aligned}
T^{\prime}(\underline{z}) & =p \underbrace{\left(\int_{p \underline{\underline{z}}}^{\infty}(p h(0)-g) f(g) d g\right)}_{(*)} w(p \underline{z})+p(p h(0)-p \underline{z}) f(p \underline{z}) \int_{p \underline{\underline{z}}}^{\underline{z}} w(y) d y \\
& =p \underbrace{p(1-F(p \underline{z}))(p h(0)-v(\underline{z}))}_{(* *)} w(p \underline{z})+p(p h(0)-p \underline{z}) f(p \underline{z}) \int_{p \underline{\underline{z}}}^{\underline{z}} w(y) d y
\end{aligned}
$$

where $(*)=(* *)$ after manipulation as shown in (7). Evaluating $T^{\prime}(\underline{z})$ at $\underline{z}=y^{*}$ gives us 


$$
T^{\prime}\left(\underline{y}^{*}\right)=p\left(1-F\left(p \underline{y}^{*}\right)\right) \underbrace{\left(p h(0)-v\left(\underline{y}^{*}\right)\right)}_{=0} w\left(p \underline{y}^{*}\right)+p\left(p h(0)-p \underline{y}^{*}\right) f\left(p \underline{y}^{*}\right) \int_{p \underline{\underline{*}}}^{\underline{y}^{*}} w(y) d y
$$

which is positive. We also have $T^{\prime}(\underline{z})>0$ for all $\underline{z}<\underline{y}^{*}$ because $p h(0)>v(\underline{z})$ for all $\underline{z}<\underline{y}^{*}$. Therefore, it must be that $\underline{z}^{*}>\underline{y}^{*}$.

Third, suppose $p h(0) \geq \bar{v}$. Then, according to Proposition 1 , we have $\underline{y}^{*}=\bar{y}$. A similar logic as before shows that we have $T^{\prime}(\underline{z})>0$ for all $\underline{z}<\bar{y}$, and therefore $T(\underline{z})$ is increasing. Because we know $\lim _{\underline{z}=\bar{y}} T(\underline{z})<T(\bar{y})$ from Lemma 5, we have $\underline{z}^{*}=\bar{y}$.

\section{Proof for Corollary 4}

Suppose $p h(0)>v(k)$. It is straightforward to show that a version of Corollary 3 holds and we have $\underline{y}^{*}>k$. As $\underline{z}^{*} \geq \underline{y}^{*}$ from Proposition 2, we have $\underline{z}^{*}>k$.

\section{Proof for Proposition 3}

First, suppose $p h(0) \leq \underline{v}$. Then, according to Proposition 1 , we have $\underline{y}^{*}=0$. As $S(0)=T(0) \leq T\left(\underline{z}^{*}\right)$, it is immediate to have $S\left(y^{*}\right) \leq T\left(z^{*}\right)$.

Second, suppose $p h(0) \in(\underline{v}, \bar{v})$. Then, according to Proposition 1 , we have $\underline{y}^{*} \in(0, \bar{y})$. There are two cases:

1. See Figure 4 for this case. If $\underline{y}^{*} \in(0, p \bar{y}]$, there exists $\underline{z}^{\prime} \leq \bar{y}$ such that $p \underline{z}^{\prime}=\underline{y}^{*}$, and this $\underline{z}^{\prime}$ will improve upon $\underline{y}^{*}$ as shown below. First, under $\underline{z}^{\prime}$, the individuals with $y<p \underline{z}^{\prime}=\underline{y}^{*}$ cannot engage in the activity according to Lemma 3 . As these individuals cannot engage under $\underline{y}^{*}$ either, social welfare from this income group is the same under both requirements. Second, consider the individuals with assets $y \in\left[\underline{y}^{*}, \underline{z}^{\prime}\right)$. Under $\underline{z}^{\prime}$, any such individual who engages in the activity will choose $x=0$ and bear expenses of $p \underline{z}^{\prime}$ according to Lemma 3. Under $\underline{y}^{*}$, the same individual chooses $x=0$ and bears expenses of $p y$. Since $p y<p z^{\prime}$, some individuals engage under $\underline{y}^{*}$ but not under $\underline{z}^{\prime}$ when $p y<g<p \underline{z}^{\prime}$, and these individuals must lower social welfare under $y^{*}$ because $g<p h(0)$. As other individuals behave in the same way under both requirements, social welfare from this income group is lower under $\underline{y}^{*}$. Third, consider the

Figure 4. CASE FOR $\underline{y}^{*} \in(0, p \bar{y}]$

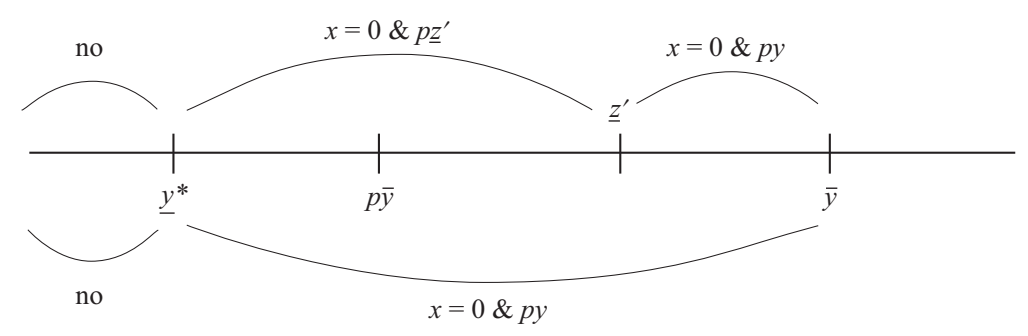


individuals with assets $y \in\left[\underline{z}^{\prime}, \bar{y}\right)$. They behave in the same way because they choose $x=0$ and bear costs of $p y$ under both requirements. Hence social welfare from this income group is the same under both requirements. Finally, the individuals with assets $y \geq \bar{y}$ exert optimal care and bear full cost of their activity under both $\underline{y}^{*}$ and $\underline{z}^{\prime}$, generating the same level of social welfare under both requirements. In sum, social welfare is lower under $\underline{y}^{*}$ than under $\underline{z}^{\prime}$. Therefore, social welfare under $\underline{y}^{*}$ must be lower than under $\underline{z}^{*}$.

2. See Figure 5 for this case. If $\underline{y}^{*} \in(p \bar{y}, \bar{y})$, we should have $\underline{y}^{*}>p \underline{z}^{*}$ because $\underline{z}^{*} \leq \bar{y}$. Thus there exists a range of $y$ (i.e., $y \in\left[p \underline{z}^{*}, \underline{y}^{*}\right)$ ) such that the individual cannot engage under $\underline{y}^{*}$, generating 0 social welfare for this group, and engages with $x=0$ under $\underline{z}^{*}$, generating for this group social welfare of

$$
\begin{aligned}
& \int_{p \underline{\underline{z}}}^{\underline{\underline{y}}^{*}}\left(\int_{p \underline{\underline{z}}}^{\infty}(g-p h(0)) f(g) d g\right) w(y) d y \\
& =\underbrace{\left(1-F\left(p \underline{z}^{*}\right)\right)}_{>0}\left(v\left(\underline{z}^{*}\right)-p h(0)\right) \underbrace{\int_{p \underline{z}^{*}}^{\underline{y}^{*}} w(y) d y}_{>0} .
\end{aligned}
$$

Because $p h(0) \in(\underline{v}, \bar{v})$, according to Proposition 1, we have $p h(0)=v\left(\underline{y}^{*}\right)$. Since $v(\cdot)$ is increasing and we have $\underline{y}^{*}<\underline{z}^{*}$ from Proposition 2, we have $v\left(\underline{z}^{*}\right)>p h(0)$. Thus (11) is positive, and therefore social welfare from this income group is higher under $\underline{z}^{*}$.

Now consider $y \in\left[\underline{y}^{*}, \underline{z}^{*}\right)$. Under $\underline{z}^{*}$, any such individual who engages in the activity will choose $x=0$ and bear expenses of $p \underline{z}^{*}$ according to Lemma 3. Under $\underline{y}^{*}$, the same individual chooses $x=0$ and bears expenses of $p y$. Since $p y<p \underline{z}^{*}$, some individuals engage under $\underline{y}^{*}$ but not under $\underline{z}^{*}$ when $p y<g<p \underline{z}^{*}$, and these individuals must lower social welfare under $\underline{y}^{*}$ because $g<p h(0)$. As other individuals behave in the same way under both requirements, social welfare from this income group is higher under $\underline{z}^{*}$. Since all other income groups behave in the same way under both requirements, we conclude that social welfare is higher under $\underline{z}^{*}$.

Finally, suppose $p h(0) \geq \bar{v}$. Then we have $\bar{y}=\underline{y}^{*}=\underline{z}^{*}$ according to Proposition 2. Thus from (1) and (3) we have

Figure 5. CASE FOR $y^{*} \in(p \bar{y}, \bar{y})$

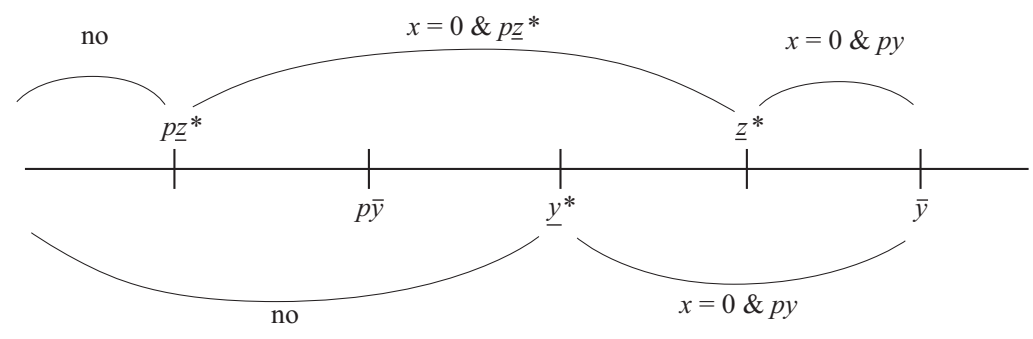




$$
\begin{aligned}
& S(\bar{y})=\int_{\bar{y}}^{\infty}\left\{\int_{x^{*}+p h\left(x^{*}\right)}^{\infty}\left(g-\left(x^{*}+p h\left(x^{*}\right)\right)\right) f(g) d g\right\} w(y) d y \\
& T \bar{y})=\int_{p \bar{y}}^{\infty}\left\{\int_{x^{*}+p h\left(x^{*}\right)}^{\infty}\left(g-\left(x^{*}+p h\left(x^{*}\right)\right)\right) f(g) d g\right\} w(y) d y
\end{aligned}
$$

which shows $S(\bar{y})<T(\bar{y})$.

\section{REFERENCES}

Abraham, K.S. (2010), Insurance Law and Regulation, Foundation Press, 5th ed.

Beard, T. (1990), "Bankruptcy and Care Choice," RAND Journal of Economics 21, pp.626-634.

Bisso, J.C. and A.H. Choi (2008), "Optimal Agency Contracts: The Effect of Vicarious Liability and Judicial Error," International Review of Law and Economics 28, pp.166-174.

Boyd, J. (2001), "Financial Responsibility for Environmental Obligations: Are Bonding and Assurance Rules Fulfilling Their Promise," Discussion Paper No. dp-01-42, Resources for the Future.

Boyd, J. and D.E. Ingberman (1994), "Noncompensatory Damages and Potential Insolvency," Journal of Legal Studies 23, pp.895-910.

Boyd, J. and D.E. Ingberman (1999), "Do Punitive Damages Promote Deterrence?," Internatioinal Review of Law and Economics 19, pp.47-68.

Boyer, M. and J.-J. Laffont (1997), "Environmental Risks and Bank Liability," European Economic Review 41, pp.1427-1459.

Cohen, A. and R. Dehejia (2004), "The Effect of Automobile Insurance and Accident Liability Laws on Traffic Fatalities," Journal of Law and Economics 47, pp.357-393.

Dari-Mattiacci, G. and G. De Geest (2005), "Judgment Proofness under Four Different Precaution Technologies," Journal of Institutional and Theoretical Economics, pp.38-56.

Dari-Mattiacci, G. and G. De Geest (2006), "When Will Judgment Proof Injurers Take Too Much Precaution?," International Review of Law and Economics 26, pp.336-354.

Dari-Mattiacci, G. and E. Langlais (2012), "Social Wealth and Optimal Care," International Review of Law and Economics 32, pp.271-284.

Dari-Mattiacci, G. and B.M. Mangan (2008), "Disappearing Defendants versus Judgment-Proof Injurers," Economica 75, pp.749-765.

De Geest, G. and G. Dari-Mattiacci (2007), "Soft Regulators, Tough Judges,” Supreme Court Economic Review 15, pp.119-140.

Dobbyn, J.F. and C.C. French (2016), Insurance Law, West Academic Publishing, 5th ed.

Freixas, X. and J.-C. Rochet (2008), Microeconomics of Banking, MIT press, 2nd ed.

Friehe, T. (2008), "On Judgment Proofness in the Case of Bilateral Harm," European Journal of Law and Economics 26, pp.175-185.

Ganuza, J.J. and F. Gómez (2008), "Realistic Standards: Optimal Negligence with Limited Liability," Journal of Legal Studies 37, pp.577-594.

Ganuza, J.J. and F. Gómez (2011), "Soft Negligence Standards and the Strategic Choice of Firm Size," Journal of Legal Studies 40, pp.439-466.

Hiriart, Y. and D. Martimort (2006), "The Benefits of Extended Liability," RAND Journal of 
Economics 37, pp.562-583.

Jerry, R.H. and D.R. Richmond (2012), Understanding Insurance Law, LexisNexis, 5th ed.

Jost, P.-J. (1996), "Limited Liability and the Requirement to Purchase Insurance," International Review of Law and Economics 16, pp.259-276.

Kahane, Y. (1977), "Capital Adequacy and the Regulation of Financial Intermediaries," Journal of Banking and Finance 1, pp.207-218.

Kim, D. and A.M. Santomero (1988), "Risk in Banking and Capital Regulation," Journal of Finance 43, pp.1219-1233.

Landes, W.M. and R.A. Posner (1984), "Tort Law as a Regulatory Regime for Catastrophic Personal Injuries," Journal of Legal Studies 13, pp.417-434.

Lewis, T.R. and D.E.M. Sappington (1999), "Using Decoupling and Deep Pockets to Mitigate Judgment-Proof Problems," Internatioinal Review of Law and Economics 19, pp.275-293.

Miceli, T.J. and K. Segerson (2003), "A Note on Optimal Care by Wealth-Constrained Injurers," International Review of Law and Economics 23, pp.273-284.

Pitchford, R. (1995), "How Liable Should a Lender Be? The Case of Judgment-Proof Firms and Environmental Risk," American Economic Review 85, pp.1171-1186.

Polborn, M.K. (1998), "Mandatory Insurance and the Judgment-proof Problem," International Review of Law and Economics 18, pp.141-146.

Rochet, J.-C. (1992), "Capital Requirements and the Behaviour of Commercial Banks," European Economic Review 36, pp.1137-1178.

Shavell, S. (1986), "The Judgment Proof Problem," International Review of Law and Economics 6, pp.45-58.

Shavell, S. (2000), "On the Social Function and the Regulation of Liability Insurance," The Geneva Papers on Risk and Insurance, Issues and Practice 25, pp.166-179.

Shavell, S. (2005), "Minimum Asset Requirements and Compulsory Liability Insurance as Solutions to the Judgment-Proof Problem," RAND Journal of Economics, pp.63-77.

Summers, J. (1983), "The Case of the Disappearing Defendant: An Economic Analysis," University of Pennsylvania Law Review 132, pp.145-185.

Sykes, A.O. (1984), “The Economics of Vicarious Liability," Yale Law Journal 93, pp.12311280. 